\title{
Review of general surgery 1974
}

\author{
HAROLD ELLIS \\ D.M., M.Ch., F.R.C.S.
}

Surgical Unit, Westminster Hospital, London SW1

\section{Introduction}

In this year's review, the author, once again, has selected those subjects which he has found of personal interest and appeal during the year. Cholecystectomy is one of the commonest major general surgical operations and, therefore, its post-operative complications are extremely important in the hospital community. Acute pancreatitis continues to be a somewhat mysterious condition which remains a clinical, diagnostic, and therapeutic challenge. There is evidence that renal calculi are being seen with ever increasing frequency in the Western World and there is naturally considerable interest in their diagnosis and management. New methods of investigation continue to make the study of deep vein thrombosis and pulmonary embolism an important and exciting topic. Realizing his surgical limitations, the surgeon is anxious to learn about new concepts of prophylaxis, early diagnosis and treatment in malignant disease; a number of interesting papers are discussed in this area. Surgeons are paying increasing attention to the local and systemic factors which are of importance in the healing of their gastro-intestinal anastomoses and much work of interest has been reported in this field. We report new studies in the management of patients with extensive burns and we conclude with one or two eye-catching papers which have been published during the year.

\section{Complications following cholecystectomy}

Cholecystectomy is one of the commonest major elective (and sometimes emergency) operations performed in the Western World. It is regarded by many practitioners as a safe and straightforward procedure, and, indeed, so it is in the majority of cases; thus Bremner et al. (1974) report no mortality in 207 consecutive elective cholecystectomies. Yet, because of the large number of patients involved, even a small proportion of complications and disasters means that a relatively large number of such problems will be encountered in any major hospital. In addition to the complications of any abdominal operation-wound infection, post-operative pulmonary collapse, deep vein thrombosis and pulmonary embolism - there is a wide range of mishaps which are specific to this procedure.
Ellis and Adair (1974) reviewed fifteen consecutive patients with bile peritonitis encountered over the past 10 years at Westminster Hospital, seven of whom were post-operative. One of these followed a week after a routine cholecystectomy and the site of leakage was not found at subsequent laparotomy. Four resulted from cholecystectomy with exploration of the common bile duct and T-tube drainage, and two followed exploration of the common bile duct, there having been a previous cholecystectomy. There were two deaths in these seven patients.

The overall mortality of bile peritonitis is high, but this appears related to age of the patient, the length of history of peritonitis and the presence of intercurrent disease rather than any specific toxicity of bile within the peritoneal cavity. Keighley et al. (1974) point out the hazards associated with the presence of infected bile at the time of cholecystectomy. Among 150 such operations, forty-nine patients had infected bile. Of forty-one having exploration of the common bile duct, twenty-three $(56 \%)$ had infected bile at the time of operation and this rose to thirty-one patients $(75 \%)$ during subsequent $\mathrm{T}$-tube drainage. Eleven emergency cholecystectomies all had infected bile and sixteen of eighteen patients operated on with jaundice at the time of surgery also had infected bile. Wound infection occurred in $40 \%$ of patients who had T-tube drainage, compared with only $15 \%$ where exploration and drainage of the duct was not required. No less than ten patients were found to have bacteraemia and three of these suffered from endotoxic shock with renal failure. Two of these three patients died and comprised the only deaths in the whole series.

The situation which is almost as distressing to the surgeon as to the patient is for residual stones to be found in the common bile duct in post-operative $\mathrm{X}$-rays after the surgeon has explored the bile duct and believes he has completely cleared it. The extent of this problem is demonstrated by Glenn (1974) who collected reports of no less than 16,700 gall bladder operations from five centres; $24 \%$ of these operations were accompanied by exploration of the common bile duct. Of these, $46 \%$ were found to have stones in the duct. Residual stones were found in $1.1 \%$ of those operated upon and in $4.3 \%$ of those 
undergoing common bile duct exploration. There is no doubt that the frequency of this complication can be reduced to a bare minimum by meticulous cholangiography at the time of operation. This will not only lessen the number of unnecessary explorations of the bile ducts, but also ensure, as far as humanly possible, that no stones are left behind. It is always a source of amazement to me that there are still surgeons who deny that this simple technique is mandatory.

A teasing problem is to demonstrate stones in the intrahepatic bile ducts and then be unable to remove them using conventional forceps. A recently devised instrument is the Storz choledochoscope. This is an instrument which is only $60 \mathrm{~mm}$ in diameter, bent to a right angle, which can be passed through the incision in the common bile duct. The stones in the intrahepatic ducts can be visualized and removed by means of a Fogarty balloon catheter (Warshaw and Bartlett, 1974).

If stones are found on the post-operative T-tube cholangiogram, what procedures are then advisable? It is first worth repeating the X-rays because artefacts produced by air bubbles, blood clot or debris may mimic stones. Way (1973) advises leaving the T-tube for 4-6 weeks if the stones are small in the hope that these will pass spontaneously and claims that this may happen in $10-20 \%$ of patients. If the stones persist, then naturally the surgeon is loath to reexplore and there has been recent increasing interest in conservative measures to get rid of the calculi. A large number of different agents have been used to infuse through the T-tube. Heparin has had some vogue but there seems little evidence that this has any specific effect, indeed, Romero and Butterfield (1974) carried out extensive in vitro experiments with gall stones incubated in bile and heparin and were unable to demonstrate either dissolution or fragmentation of the calculi. Catt and his colleagues (1974) considered simple lavage alone to be highly effective. They report ten patients with retained stones after common bile duct exploration in which the following simple technique was used: propantheline bromide was given intramuscularly $30 \mathrm{~min}$ before flushing of the ducts, which was carried out with 1 litre of normal saline containing $40 \mathrm{ml}$ of $1 \%$ lignocaine. The infusion was commenced slowly by means of a drip and then allowed to flow as fast as possible. Three daily washouts were given and the cholangiogram then repeated. The technique was successful in six of the ten cases, the largest stone being $10 \mathrm{~mm}$ in diameter on X-ray. Nine of the patients had positive bile culture and eight of these had pyrexia after lavage. Antibiotics were not used during this technique but the authors consider that perhaps they should have been! There has been much interest in the use of bile salts as lavage fluid;
Lansford and his colleagues (1974) used an infusion of sodium cholate at a rate of $30 \mathrm{ml} / 1 \mathrm{hr}$ for a maximum of 10 days. They had five successes in six patients, the stones being passed within a few days. Two of their patients had transient episodes of pancreatitis as the stone impacted in the ampulla. The diarrhoea associated with this technique was controlled by means of cholestyramine. In vitro experiments by Lahana, Bonorris and Schoenfield (1974) suggest that a heparin/sodium cholate combination may be more effective. Semb and his colleagues (1974) successfully removed large residual cholesterol stones in two patients by installation of $5 \mathrm{ml}$ of chloroform warmed to $40^{\circ} \mathrm{C}$. In the first, one treatment was sufficient; in the second, the installation was repeated on a second occasion.

The development of the image intensifier has enabled radiologists to develop new techniques for removal of retained calculi under X-ray control. Burhenne (1974) records 120 patients with residual biliary tract stones in whom he has introduced a steerable catheter along the track of the T-tube drain under X-ray control and through which he has passed a Fogarty balloon catheter or Dormia basket. He was able to remove the stones in no less than 115 of these cases. In the five failures, one had a track which was too tortuous, in two the track was too narrow, one had a calculus lodged in a pocket adjacent to the ampulla and one was operated upon without being submitted to a proposed second attempt at removal. He has been able to fragment and remove stones as large as $3 \mathrm{~cm}$ in diameter. Complications were few-three patients had septicaemia which responded to ampicillin and one sustained a short false passage in the abdominal wall which gave no further problem.

If all conservative means fail, then the duct must obviously be re-explored and the second operation may be helped by the rigid fibre-optic choledochoscope. Of course, if persistent obstruction, pain or cholangitis occur, then exploration may have to be carried out at an early stage, rather than waiting on these more conservative measures (Bartlett, Warshaw and Ottinger, 1974).

It is worth noting that not every episode of jaundice following a cholecystectomy must necessarily mean residual stones or stricture. Peel and Ritchie (1974) have encountered two examples of Gilbert's syndrome in sixty-seven consecutive male cholecystectomies. This is a syndrome characterized by intermittent unconjugated hyperbilirubinaemia accompanied by jaundice and which is due to a congenital metabolic defect; if not recognized this may lead to re-operation in patients following cholecystectomy.

A bizarre complication of cholecystectomy has been reported by Halvorsen and Semb (1974). This 
occurred in a male patient aged 77 who had his right hepatic artery injured during removal of the gall bladder. The artery was repaired but 6 months later the patient had a fatal haematemesis. At postmortem there was a false aneurysm of the right hepatic artery measuring $4 \mathrm{~cm}$ in diameter which had ruptured into the duodenum.

\section{Acute pancreatitis}

Acute pancreatitis remains a fascinating and difficult clinical problem from the points of view of aetiology, diagnosis and treatment. In a useful review of 140 cases seen at the Royal Infirmary, Glasgow, Imrie (1974a), found that 127 were primary (with a mortality of $18.9 \%$ ) and fourteen were due to blunt trauma or followed abdominal surgery, with a mortality of $46 \%$. In $33 \%$ of the patients there was no aetiological factor to be determined. Fifty per cent of the patients had gall stones and $12 \%$ were alcoholics, a figure that is particularly high in Glasgow when compared with statistics from other parts of the country. Six cases occurred in pregnancy or postpartum and four of these patients had gall stones. Mortality was closely related to age; under the age of 50 this was $5.5 \%$ but in the seventy-nine patients over the age of 50 the mortality rose to $29 \%$. Acute renal failure occurred in twenty patients and fifteen of these died. Pseudocyst formation occurred in fifteen patients and no less than $37 \%$ of the series (fifty-two patients) had recurrent attacks of pancreatitis, a surprisingly high figure. Wyatt (1974) points out that a surgical unit serving a population of 100,000 is likely to admit five patients a year with acute pancreatitis. He finds that the only reasonable constant clinical finding in this condition is pain, and notes that one peculiar feature is that the patient, despite his shocked state, may insist on sitting rather than lying; this presumably relaxes the tension in the stretched retro-peritoneal tissues and gives a measure of relief. The serum amylase estimation remains the most important clinical investigation; raised levels are maximal 5-12 $\mathrm{hr}$ after the onset of symptoms and then fall rapidly. The urinary amylase is raised for a longer period. It is important to note that the amylase level is often raised in perforated peptic ulcer, acute cholecystitis, intestinal strangulation, ruptured aortic aneurysm and particularly in afferent loop obstruction after gastrectomy. A lowered serum calcium below $7 \mathrm{mg} \%$ is associated with a poor prognosis and the appearance of methaemalbumin indicates the presence of haemorrhagic pancreatitis from the action of proteolytic enzymes on haemoglobin, again with a correspondingly poor prognosis.

Among the rarer aetiological factors in acute pancreatitis are hyperparathyroidism (only one example in the 590 collected cases reported by
Trapnell, 1974), steroid therapy and a wide range of other drugs, including the chlorothiazides, azathioprine and the contraceptive pill. Seven of Trapnell's 590 patients had mumps, and recently Feldstein et al. (1974) reported the first confirmed example of haemorrhagic pancreatitis with subsequent pseudocyst formation in a child with mumps. In this patient, a Negro child aged 4, the diagnosis of haemorrhagic pancreatitis was confirmed at operation and the mumps documented by rising serum titres.

An interesting clinical observation, apparently not previously recorded, is presented by Sankaran, Lucas and Walt (1974). In a group of forty-two patients with acute alcoholic pancreatitis in Detroit no less than forty were observed to develop a transient hypertension which fell to normal when the patient recovered clinically and biochemically. This did not occur in other acute abdominal emergencies that were carefully observed. The renin level was not raised in these patients and the authors postulate the production of some as yet unidentified vasoactive substance in this condition.

Imrie (1974b) comments on the facial flushing in the absence of pyrexia which was noted in no less than thirty-eight $(44 \%)$ of eighty-six patients admitted with acute pancreatitis. In most it was seen within $12 \mathrm{hr}$ of admission but might be delayed to up to $36 \mathrm{hr}$. The flushing usually persisted for a day but a 'beacon-like appearance' might last a much shorter time. The sign has little prognostic significance but may help in diagnosis. Imrie postulates that it may be related to the release of vasoactive peptides into the peripheral blood.

The treatment of acute pancreatitis remains controversial, since the mortality and morbidity of different series vary quite widely and because, to date, very few clinically controlled trials have been reported. Feller et al. (1974) studied eighty-three cases with severe acute pancreatitis out of a total of 200 patients with this condition at the Montreal General Hospital. There were twelve deaths in this group, five due to abscess or pseudocyst, four due to irreversible shock (all over the age of 75), two from haemorrhage and one from hepatic failure. The main complications were severe nutritional depletion, abscess or acute pseudocyst formation, respiratory failure and upper gastro-intestinal bleeding. General treatment included gastric suction, intravenous feeding, analgesia supplemented by epidural block, penicillin and kanamycin therapy, peritoneal irrigation, and respiratory care, with endotracheal intubation or tracheostomy if necessary. They advise surgery if the diagnosis is in doubt, if the patient's condition is deteriorating and if there is abscess or pseudocyst formation. At laparotomy the gall bladder is removed or decompressed if diseased, the 
common bile duct drained and a gastrostomy performed for later feeding. Trapnell (1974) advises laparotomy if a firm diagnosis of acute pancreatitis cannot be made or where rapid deterioration is taking place in spite of adequate resuscitation; provided that the laparotomy is properly conducted, mortality is not increased. If the patient is found to have gallstones, cholecystectomy should be performed and cholecystostomy reserved for patients who are very ill. An operative cholangiogram should be performed and if stones are demonstrated in the common bile duct, these should be removed without interfering with the region of the ampulla. If the patient does not have biliary tract disease, Trapnell's studies show quite conclusively that biliary tract surgery will not confer any benefit.

In severely ill patients there is the question of whether prognosis might be improved by carrying out a total or near total pancreatectomy in order to remove the sloughing pancreas. Norton and Eiseman (1974) point out that the mortality of haemorrhagic pancreatitis is often more than $50 \%$. They report four cases in which a subtotal distal pancreatectomy was performed and they note that this was a relatively easy procedure; the gland dissects out readily from the adjacent oedematous tissues and many small vessels are thrombosed, so that bleeding is not a serious problem. In three of their cases, all alcoholics, survival occurred, although one developed diabetes. The remaining patient, in whom haemorrhagic pancreatitis complicated cholecystectomy, died as a result of leakage from a gastroenterostomy performed at the same time. Francillon et al. (1974) studied fourteen patients with acute necrosing pancreatitis, twelve primary and two postoperative (following a gastrectomy and a sphincterotomy); seven were alcoholics. In ten of these they performed what they term 'necrosectomy'digital removal of all sloughing necrotic pancreatic tissue. Five of these patients survived, although with pancreatic fistulae that persisted for up to 3 months. In the four remaining patients, the necrotic pancreas was merely drained and all four died.

A number of drug treatment regimes have been the subject of considerable recent interest. Hallberg and Theve (1974) report twenty patients with acute pancreatitis treated only by intravenous insulin and glucose. Twenty units of insulin per litre of $5 \%$ glucose were given at a rate of $300-400 \mathrm{ml}$ in the first hour. Fifteen became free from pain within a few hours in spite of no analgesics having been given. They found that there was less drop in the blood glucose level in their patients with acute pancreatitis than in matched normal controls. This extraordinary claim certainly warrants further investigation.

Recently it has been suggested that glucagon may be beneficial in the treatment of this condition and the Medical Research Council is setting up a full trial to study this (Welbourn and Cox, 1974).

Perhaps the most interesting substance in this context is trasylol (aprotenin) which is a proteolytic enzyme inhibitor. Although this has been available for some 15 years its effectiveness has never been firmly established. Recently Trapnell and his colleagues (1974) have reported a blind clinical trial on fifty-three patients treated with trasylol and fifty-two matched controls. The patients were graded into mild, moderate and severe groups. All were treated in a standard manner with Pro-Banthine, cytamycine and supportive therapy. In the trasylol group there were four deaths $(7.5 \%)$ compared with thirteen deaths in the controls $(25 \%)$, statistically a significant difference. These encouraging results naturally create considerable interest (Leading article, British Medical Journal, 1974) and certainly warrant further controlled trials in other centres (Imrie, Whyte and Blumgart, 1974).

\section{Renal calculi}

It is interesting that stone in the bladder, which was once epidemic throughout Europe and which was seen quite commonly in young children, is now becoming quite rare in the Western World. Stone in the kidney and ureter, however, remains a common phenomenon and, indeed, seems to be on the increase. In many cases the aetiology remains unknown, although careful study may often reveal some precipitating factor. Morrison (1974) has reported a study of 322 patients with renal calculi in New Zealand. The history revealed a positive family history in forty-one, prolonged immobilization in forty-seven, urinary infection in forty-seven, a period of domicile in the tropics in thirty-seven, a dyspeptic history in ninety-one, and a heavy analgesic intake in seventeen. Metabolic studies demonstrated idiopathic hypercalciuria in seventy-three patients, hyperparathyroidism in five (three with a parathyroid tumour), uric acid stones in twelve, renal tubular acidosis in twelve and cystinuria in two patients. In seventy patients no possible cause could be demonstrated, but the author points out that a stone may manifest itself years after its formation, by which time the original aetiological factor may be impossible to demonstrate. Sutor, Wooley and Illingworth (1974) have carried out a meticulous chemical study of 856 stones collected from various centres in the United Kingdom. Three-hundred-and-twentyfive of the stones contained a single crystalline substance, of which by far the commonest was calcium oxalate. Of the mixed stones, the commonest combination was calcium oxalate and phosphate.

Many stones in the upper urinary tract are very small, pass easily after a few episodes of colic, and 
pose little or no problem for the surgeon. Most troubles come from the large laminated calculus which is often associated with urinary infection and obstructive uropathy. Of 190 renal stones which required operation, Blandy (1974) found that eightyfive were large branched calculi and infection was present in $71 \%$. These days it is usually possible to save the kidney and indeed in no instance in this series was nephrectomy required. In seventeen cases, calculi or fragments were left behind after removal of the main mass. In four patients these passed spontaneously, in nine they remained unchanged and in only four did they form the basis for new calculus formation, so it is evident that small residual particles of stone are only one among many factors that may be responsible for the recurrence of large urinary calculi after surgery.

In operations for large renal calculi, there are times when renal hypothermia can be useful, especially when a small fragment is left behind in an outlying calyx or when a large mushroom extension of the main stone has to be removed through a thick layer of overlying renal tissue. Generally surgeons have cooled the kidney with ice slush, but it is tedious to prepare this and to ensure both sterility and the right consistency. Surface cooling coils are associated with the risk that tissue in direct contact with the coil may become frostbitten. Marshall and Blandy (1974) describe a very simple method which uses only standard equipment. Cold normal saline is led through an ordinary transfusion set to a blood-warming coil immersed in unsterile icesalt mixture and then via an extension tube to the kidney exposed at operation. Renal temperature is measured with a thermocouple placed $2 \mathrm{~cm}$ below the surface of the kidney. A split plastic sheet is arranged round the kidney so as to form a well into which the cold saline is run at a temperature of $6-7^{\circ} \mathrm{C}$. The renal artery is occluded with a bulldog clamp. If a staghorn calculus is removed, as much as possible is taken out through an extended pyelolithotomy and the cold saline is then run directly into the opened renal pelvis. It takes $10 \mathrm{~min}$ to achieve cooling of the kidney to $20^{\circ} \mathrm{C}$. With this technique the authors have used periods of ischaemia for up to $55 \mathrm{~min}$.

Emergency surgery for renal stone is discussed by Leong, Yu and Ong (1974) from Hong Kong. This is indicated when there is anuria or severe oliguria due to obstruction of the solitary kidney or the sole functioning kidney, or if there is acute pyonephrosis due to the onset of infection in an obstructed kidney. In a series of 437 patients with upper urinary calculi, only nineteen $(4.4 \%)$ were admitted for emergency surgery. In those patients with anuria and an associated high blood urea, cystoscopy and retrograde catheterization was carried out under local anaesthesia, which may enable the obstruction to be bypassed and temporary drainage to be established. If this failed, dialysis was carried out followed by early surgical removal of the obstruction. Patients with no, or with minimal, urea retention were operated on as emergencies and usually had a temporary proximal diversion, using either a T-tube ureterostomy or a nephrostomy. In the eleven patients with acute pyonephrosis the primary aim of treatment was to establish drainage of the infected kidney in an attempt to prevent complete renal destruction. If the stone was within easy reach it was removed, otherwise temporary drainage was carried out. In cases where the kidney was completely destroyed, nephrectomy was performed after establishing that there was adequate function of the contra-lateral kidney.

In his Hunterian lecture, Sreenevasan (1974) discusses the difficult problems presented by bilateral renal calculi. These were found in 114 of 1070 cases of urinary stone treated in Kuala Lumpur $(10.7 \%)$. Renography is used to assess which is the better kidney, and operation is carried out on this side first. If the blood urea is raised to between 50 and $150 \mathrm{mg}$ but there is a reasonable urinary output, intravenous fluids and blood transfusion are given; when the urine output is low, the patient is put on frusemide. If the patient fails to improve and his blood urea continues to rise, dialysis is performed. Once the condition of the patient has stabilized, surgery is carried out on the better functioning kidney. When the blood urea is raised above $150 \mathrm{mg}$, the general condition is first improved by dialysis. Using this regime on the last 100 cases of bilateral renal calculi, there were three deaths, two of which were unrelated to the renal tract. Recurrent renal calculi formed in fifteen of these patients. The problem of recurrent stones is also considered by Powis et al. (1974) in a retrospective survey of 305 patients with proved urinary calculi, of whom fifty-four had bilateral stones and 142 required surgical removal of one or more calculi. No less than 110 patients had more than one episode of calculous disease $(36 \%)$. The pick-up of underlying causation in these cases was rather disappointing; only two patients had hypercalcaemia and of these one had a parathyroid tumour. Abnormalities of the renal tract, hydronephrosis etc. were found in a total of nineteen patients, six of whom had multiple episodes. Routine biochemical studies and radiology was, therefore, of little help in picking up those patients who were liable to develop recurrent calculous disease. These authors conclude that it seems reasonable that patients without complications could be discharged from follow-up after their initial episode had settled and investigation is complete. Such patients could be asked to return when and if symptoms recur as 
at that stage further radiological examination has something to offer.

Once a stone has entered the ureter, the probability that it will pass spontaneously is closely linked to its size. Below $4 \mathrm{~mm}$, over $90 \%$ of the stones in the lower ureter and $80 \%$ of stones in the upper ureter will pass spontaneously, whereas at 4-6 mm only $50 \%$ of the stones in the lower ureter and $20 \%$ of those in the upper ureter will pass. When the stone is larger than this the chances of spontaneous passage are small. When first seen, nearly $80 \%$ of stones are in the lower third of the ureter. If the patient remains completely symptom-free after his first attack of renal colic and if there is no serious obstruction on X-ray, it is obviously reasonable to wait and watch. However, many patients are not symptom-free and may undergo repeated episodes of pain. Walsh (1974) argues that such patients should be offered the relief of endoscopic extraction of the calculus at an early stage. For this he usually uses the Dormia basket, which is an ingenious wire snare passed along a ureteric catheter via an operating cystoscope. The closed basket is passed above the stone, the basket opened, drawn down and hopefully entangles the calculus and removes it. It should not be used on large stones and should rarely be used above the lower third of the ureter. It should not be employed when there is a combination of ureteric obstruction and acute infection. If the basket sticks above the intramural ureter it is probable that there 'is a fold of ureteric mucosa caught between the basket and stone. In this situation it is imperative not to apply the slightest force, but to proceed to open surgery. When the basket successfully gathers the stone, it will commonly stick in the intramural ureter, but provided that at least half of the basket is visible in the bladder it is perfectly safe to pull as hard as is necessary to get the stone out. If the stone presents in the intramural ureter and can be seen at cystoscopy, either bulging into the bladder or protruding out of the ureteric orifice, Walsh advises a transurethral uretero-lithotomy, cutting directly onto the stone with a knife electrode. In a consecutive series of 126 cases of stone in the ureter where the Dormia basket was used, the author reports that the stone was extracted immediately in eighty-two $(65 \%)$. In twenty-four more patients the stone passed spontaneously in a period of up to 6 weeks. In two instances the basket stuck and immediate open surgery was performed. In eleven further patients elective surgery was carried out and the fate of seven other patients is not recorded.

\section{Venous thrombosis and pulmonary embolism}

Interest in postoperative venous thrombosis and pulmonary embolism remains intense and the flood of publications in this field continues, and for very good reasons. In the first place, pulmonary embolism persists as one of the important unsolved problems in the post-operative period, in the second place advances in techniques of investigation have given us far more accurate methods of determining the presence of venous thrombosis and of pulmonary embolism, and finally considerable efforts are now being made in developing efficient methods of both prophylaxis and treatment in this field. Negus and Rickford (1974) give a useful summary of the three available special investigations for venous thrombosis in the lower limb. Venography enables us to determine the site of the thrombus and to assess whether this is fixed or floating freely and dangerously within the lumen of the vein. The investigation cannot be carried out repeatedly and is, therefore, not of value as a means of continued monitoring. The radioactive iodine-labelled fibrinogen scan technique is extremely accurate in the lower thigh and calf (indeed, it may be too accurate in demonstrating what may be clinically insignificant calf thrombi). The investigation can be repeated at frequent intervals but, because of interference from radioactive iodine excreted into the urine and hence picked up on the scan as scatter from the bladder, scanning of the iliac vessels and upper parts of the femoral veins cannot be carried out. In this very zone the use of the Doppler ultrasound probe is extremely valuable in indicating whether there is a total or partial block in these major vessels. The use of these techniques has demonstrated in many trials the surprisingly high incidence of deep vein thrombosis in between 30 and $60 \%$ of adult patients undergoing major general surgery, $18 \%$ of gynaecological cases, $54 \%$ of orthopaedic patients and $50 \%$ of urological cases (Skillman, 1974). Certain groups particularly at risk can also be defined and these include elderly, obese patients suffering from malignant disease, with varicose veins and with a previous history of deep venous thrombosis and pulmonary embolism. For some strange reason, white patients are far more prone to this condition than some other races, for example, the Malaysians (Cunningham and Young, 1974) and the Sudanese (Hassan, Rahman and Rahman, 1974). Obviously further world-wide studies are needed on this topic, since Joffe (1974), in Cape Town, was unable to demonstrate any difference in incidence or distribution of thrombosis between his white, black and Asian patients.

Until recently it was thought that the oedema which is so often seen after arterial reconstructive surgery in the lower limb was due to a postoperative venous thrombosis. However, Myhre, Storen and Ongre (1974) have now carried out a venographic study of twenty-five patients with oedema of the lower limb after successful arterial reconstruction. Deep vein thrombosis was found in only two of these 
cases $(8 \%)$ and they attribute the low incidence of this complication to the post-reconstructive hyperaemia and increased venous flow in the operated limb. The oedema may be due to increased filtration from capillaries suddenly subjected to an increase in perfusion pressure. This study helps to emphasise that the clinical diagnosis of deep vein thrombosis is far from accurate, indeed the accuracy of diagnosis from physical signs has been quoted variously as lying between 28 and $53 \%$. Tibbutt and Gunning (1974) point out that haemorrhage into the calf may produce symptoms and signs that closely mimic those of deep vein thrombosis. They have observed a sign in four patients which has apparently not previously been described, a crescentic area of bruising which develops around the malleoli as a result of the blood gravitating towards the ankle; this may be of value in differential diagnosis.

\section{Prophylaxis}

The demonstration of the high incidence of postoperative calf vein thrombosis and the good evidence that the majority of major thromboses and of pulmonary emboli originate from clot propagation from the calf veins (Fossard et al., 1974b) has led to an intensive search for effective and safe prophylactic measures, which have been well reviewed by Kakkar (1974). First there are physical methods, which include intensive physiotherapy, electrical stimulation of the calf muscles, pneumatic compression and passive fiexion of the calf. Drugs may be employed which affect platelet function; with this in mind, aspirin, phenformin and ethyloestrenol have been employed without being shown to be effective, but claims for the use of intravenous dextran have been more encouraging. Finally, there has been much interest in the use of low dosage heparin given subcutaneously in a dosage level of 5000 units three times daily for 7-10 days post-operatively.

There is good evidence that most cases of deep venous thrombosis originate in the operating theatre; during this time the patient is under full relaxant anaesthesia, often with positive pressure respiration, and these abolish all the factors upon which venous return depends apart from left ventricular output (Doran, 1974). Although leg elevation and elastic stockings increase venous return from the legs, fibrinogen uptake studies show that these measures have no effect on deep vein thrombosis. However, both electrical stimulation of the calf muscles and intermittent compression of the leg are effective in reducing thrombosis, probably by emptying areas of extreme venous stasis at the valve cusps within the calf muscles (Browse et al., 1974). Several studies have now shown that a simple intermittent compression machine which squeezes the calf muscles during, and for $24 \mathrm{hr}$ after operation greatly reduces the incidence of thrombosis (Clark et al., 1974; Roberts and Cotton, 1974).

Turning now to attempts at affecting platelet function, it is disappointing that Fossard et al.(1974a) were unable to show a difference in the incidence of deep venous thrombosis in patients undergoing gynaecological surgery who received phenformin and ethyloestradiol for 3 weeks before surgery compared with controls, even though this regime shortened the dilute blood clot lysis time. These drugs obviously stimulate systemic fibrinolytic activity and it is possible that they might at least prevent extension of the clot.

The combined results of four studies using subcutaneous heparin have shown a reduction in the incidence of deep venous thrombosis on radioactive fibrinogen scanning from $26 \%$ in the controls to $3 \%$ in the treated group without significant complications (Gordon-Smith, 1974). Van Vroonhoven, van Zijl and Muller (1974) have demonstrated the superiority of low-dose subcutaneous heparin over oral anticoagulants in this respect. Naturally, the vital factor is whether this therapy actually reduces the incidence of post-operative pulmonary embolism; this is certainly suggested by the trial reported by Lahnborg et al. (1974). These authors report a double-blind trial on fifty-four control patients and fifty-eight treated cases undergoing major surgery who were followed-up with chest photoscans and chest X-rays, together with fibrinogen scanning of the legs. In the fifty-four controls there was a $36 \%$ incidence of scans suggesting pulmonary embolism compared with a $14 \%$ incidence in the treated group. The preliminary results of a multi-centre trial also indicate the efficacy of this form of therapy (Kakkar, 1974).

An interesting study that certainly warrants further trial is the report by Carter and Eban (1974) that hydroxychloroquine sulphate is an effective agent reducing deep venous thrombosis after major surgery. This agent is not an anticoagulant and is effective when given orally. When given as a preoperative loading dose of $1200 \mathrm{mg}$ in divided doses during the $24 \mathrm{hr}$ preceding operation, followed by $800 \mathrm{mg}$ daily in two divided doses until discharge from hospital, the incidence of deep vein thrombosis, as assessed by leg scanning, was reduced from a control level of $16 \%$ to $5 \%$ in the treated group in a study of 204 patients over the age of 45 subjected to non-emergency major surgery.

\section{Pulmonary embolism}

In an interesting review of the situation in France, Marion and Binet (1974) note that pulmonary embolism is the cause of death in no less than $26 \%$ of patients over the age of 40 coming to post-mortem in hospital practice. They estimate that there are 
142,000 annual deaths from this cause in the U.S.A., 21,000 in the United Kingdom, and about 20,000 in France, where there is an incidence of fatal pulmonary embolism of 55 per 100,000 operations. In a review of 1026 fatal pulmonary embolisms occurring in 1972, they found that only sixteen attempts had been made at embolectomy, of which nine were successful. In many cases there is a herald embolus before the massive embolus occurs.

Browse (1974a) considers that phlebography is essential in such cases in order to plan further management. If fresh thrombus is present, he advises thrombectomy or thrombolysis using streptokinase or urokinase. If there are multiple small peripheral thrombi, he considers it safer to ligate the vein upstream and confine the thrombus to the limb; ligation of the superficial femoral vein below the entry of the profunda vein has been sufficient for more than $80 \%$ of his cases. Further thrombosis is prevented by giving adequate heparin therapy. If regular blood tests are not possible, he advises a minimum dose of 40,000 units every $24 \mathrm{hr}$, given either continuously or 4-hourly. The effect of a single dose of heparin wears off in $4 \mathrm{hr}$ so that the common 6-hourly regime leaves the patient untreated for $8 \mathrm{hr}$ in every $24 \mathrm{hr}$ (Browse, 1974b). In the U.S.A. it is very fashionable to obstruct the vena cava (or in the case of a recent famous politician, the iliac vein) which may be ligated, plicated, clipped, or stenosed. Although in this country Gardner et al. (1974) advocate the use of a smooth silicone rubber clip in order to produce partial occlusion of the cava, this technique has never become particularly popular in the United Kingdom. More recently the Americans have experimented with a variety of ingenious umbrellas which can be placed in the cava, and Knight, Amplatz and Nicoloff (1974) have now devised a technique for introducing this apparatus by a Seldinger technique through the femoral vein. Silver (1974), however, considers that adequate heparin therapy alone is just as effective as caval interruption, both with regard to mortality and recurrence, and Ebert (1974) points out that all too often an inadequate dosage of heparin has been prescribed. He estimates that the risk of further embolism falls to the region of $2 \%$ if adequate, continuous anticoagulation is maintained.

When the pulmonary embolism is life-threatening the choice of treatment lies between what might be called conventional therapy, that is to say the use of heparin, morphia and oxygen, or thrombolytic treatment using streptokinase and other lytic agents, or pulmonary embolectomy using cardio-pulmonary bypass. Tibbutt et al. (1974) have carried out a comparison by controlled clinical trial between streptokinase and heparin in such cases, all confirmed by angiography, and consider that streptokinase is the treatment of choice. However, if the blood pressure remains below 100 and angiography shows a major pulmonary blockage then they consider embolectomy to be indicated. It is obvious, however, that this can only be carried out if the patient is in a major centre or when he can be transferred rapidly to a cardiothoracic unit.

\section{Growing points in cancer}

It is doubtful if any surgeon believes that the problems of cancer are going to be overcome by the development of bigger and better operations, so that the clinician must obviously turn to his colleagues in other disciplines in the hope that their investigations are throwing some rays of optimistic light into this otherwise gloomy subject. Our aim should naturally be prevention, and the subject of carcinogens in the environment has been ably reviewed by Magee (1974). There is still no firm proof that any form of human cancer is caused by a virus, but there is increasing evidence that some, particularly lympho-reticular tumours, may be so induced. It is suggested that not more than $10 \%$ of human tumours are caused by radiation or viruses, leaving the remainder attributable to exogenous or endogenous chemical induction. Although a very large number of chemical agents are known to produce cancer in the experimental animal, there are relatively few for which there is fairly convincing evidence for carcinogenic activity in man. These include asbestos, arsenic, chromium, nickel, benzene and some aromatic amines, coal-tar, pitch, soot, various fuel and lubricating oils, mustard gas and two parasites, Schistosoma haematobium (associated with bladder cancer) and Clonorchis sinensis (associated with tumours of the intra-hepatic bile ducts). It now seems probable that diethylstilboestrol or related compounds, used in the treatment of threatened abortions, are responsible for recently described cases of adenocarcinoma of the vagina in adolescent girls. Carcinoma of the liver has been associated with prior treatment with anabolic steroids and several cases of benign hepatoma have been reported in women taking oral contraceptive steroids. Most recent of all, angiosarcoma of the liver has been observed in workers manufacturing polyvinyl chloride. In this case the responsible agent is probably the monomer, vinyl chloride. An example of this has now been reported from Blackpool by Lee and Harry (1974) in a 71-year-old man who had been employed in the manufacture of this substance for 20 years. These authors collect nineteen previous reports from the U.S.A., Sweden and Germany in men working with vinyl chloride in periods of between 12 and 27 years. Naturally, the possibility of this material finding its way into the food and atmosphere has aroused considerable alarm and 
interest (Leading article, Lancet, 1974; Leading articles, British Medical Journal, 1947a, b), and of particular significance is that these tumours are very rare in western countries and were thus conspicuous in small numbers. If newly introduced environmental factors are currently causing increases in the incidence of more common human tumours, the relationship will be much more difficult to detect. Only a very small proportion of the components of the chemical environment have been tested in any way for their carcinogenic potential and the number of compounds is constantly being increased by the output of the chemical industry. Conventional tests for carcinogenicity usually require life-time exposure of animals to the chemical under test; they often take more than 3 years to complete and are very expensive. Screening might be more rapid and inexpensive, using mutation of micro-organisms, the unscheduled synthesis of DNA or transformation of cells in vitro. Professor McGee concludes that there are many known or potential human carcinogens and that their removal or elimination from the environment could result in a greatly decreased incidence of human cancer. An obvious example is the marked reduction in human lung cancer that follows giving up cigarette smoking.

For many years human tumours have been grown in laboratory animals as xenografts. The usual technique has been to introduce the tissue into an immunologically privileged site, such as the cheek pouch of the hamster or the anterior chamber of the eye, where it remains isolated from the host's immunological defences. Recently there has been considerable interest in the growth of human tumours in animals which have been rendered immunologically incompetent. Castro and Cass (1974) describe their technique in which mice are first thymectomized, then given a lethal dose of total body irradiation and then an intravenous injection of bone marrow cells from mice donors of the same strain. Sixty-eight per cent of human tumours implanted beneath the renal capsule of such animals were viable at 2 weeks, and many survived for 3 months or more. In some cases the tumours grew and in some it was possible to transmit the tumour to secondary hosts in up to five passages. The practical application of this interesting work has yet to be explored (Leading article, British Medical Journal, 1974c). At present, the uncertainty of 'take' is a problem, but if lines of transplantable human cancers could be established then they might be used as a laboratory system for screening of new cytotoxic drugs as well as for the study of the metabolism of therapeutic agents by human tumours.

\section{Cancer immunology}

Early high hopes that immunotherapy might produce dramatic results in the treatment of advanced malignant disease have not been sustained by clinical experience. However, interesting and encouraging work continues in this field. Feneley et al. (1974) report from Bristol on their experience in the treatment of advanced bladder cancer with sensitized pig lymphocytes. The pig is immunized by implantation of tumour fragments from the patient into its ileal mesentery. Seven days later, the patient receives an intra-arterial infusion of mesenteric lymph node cells from the donor. In seven patients this was the only treatment employed. Tumour necrosis and cessation of haematuria occurred in five instances and in two there was a decrease in tumour size. In another eleven patients this treatment was followed by an attenuated course of radiotherapy and in this group nine of the patients had tumour regression, and two patients at their time of death from widespread metastases had no evidence of bladder tumour. The infused pig lymph node cells, having been pre-immunized against the tumour, will attack the neoplastic cells after a shorter time interval than that required for the rejection of the pig cells by the patient. It is also suggested that the reaction between a sensitized lymphocyte and its specific target cell will activate the patient's macrophages, which are capable of destructive action against target cells. A controlled trial has now been instituted to compare the effect of treating matched pairs of patients either by pig cells followed by radiotherapy or by radiotherapy alone.

Perhaps of more immediate practical application is the use of sensitive immunological screening tests in cancer diagnosis, cancer screening and in the early detection of recurrent disease. Primary hepatocellular carcinoma is often difficult to diagnose and, until recently, biopsy was the only means of confirmation. The value of alpha-feto protein (A.F.P.) is now well established. This globulin is present in high concentration in fetal serum but only in small amounts thereafter. Ten years ago it was first reported as being raised in primary liver cancer, thus suggesting an immunochemical marker useful in the diagnosis of this tumour. A recent useful review by Kew (1974) points out that A.F.P. may also be raised in malignant teratoblastoma of testes and ovary, in a minority of tumours of endodermal origin (pancreas, gall bladder, lung and stomach) and in a variety of other liver diseases, including acute viral hepatitis. It has also been reported as being raised in one example of malignant lymphogranuloma and one case of Wilm's tumour. In these instances it was first suggested that the raised A.F.P. was due to rapidly regenerating hepatic tissue adjacent to liver secondaries, but examples have now been reported in the absence of metastatic deposits in the liver. Kelleher et al. (1974) record two patients with gastric carcinoma with liver 
secondaries having raised A.F.P. and note that this has been reported in a case of carcinoid of the stomach with liver deposits. Kohn and Weaver (1974) report an extensive survey from the Westminster Hospital Group of A.F.P. screening of the sera from 2225 patients, 107 of whom had primary hepatocellular carcinoma. Of these 107 patients, ninety were A.F.P.-positive, and four who were negative on screening were shown by radio-immuno-assay to have significantly raised serum A.F.P. levels. Raised levels of A.F.P. after surgery or chemotherapy indicate failure of treatment, although the converse is not true. In malignant testicular teratoma, the frequency of raised serum A.F.P. was thirty-two out of 117 cases $(27 \%)$. The frequency of false-positive findings in this study was $1.8 \%$.

Early high hopes that estimation of the plasma levels of carcino-embryonic antigen (C.E.A.) might prove to be a specific screening test for large bowel cancer have later proved disappointing. In most studies in patients with carcinoma of the colon and rectum between $30 \%$ and $60 \%$ of patients with localized disease have raised levels, while the positivity approaches $100 \%$ when dissemination has occurred. Present interest is concentrating on the use of C.E.A. assays in detection of recurrent and metastatic colo-rectal cancer. Booth et al. (1974) have used C.E.A. estimation in the preoperative assessment of colo-rectal carcinoma and have shown that a serum concentration of $100 \mathrm{ng} / \mathrm{ml}$ or more is a useful guide to the presence of metastatic disease and ultimately to poor prognosis, and to be more reliable as an index of tumour spread than either clinical examination or serum alkaline phosphatase estimation. The test has also been assessed in a group of patients attending cancer follow-up clinics after radical resection. Raised C.E.A. occurred in most of those developing recurrent disease and in several patients a rising C.E.A. level preceded clinical or biochemical evidence of recurrence. Mackay et al. (1974) have carried out C.E.A. estimations in 220 patients who had undergone apparently curative surgery for colo-rectal cancer. In a 2-year follow-up period, fifty-three patients developed recurrences or metastases and in thirty-six of these there was a sustained rise in plasma C.E.A. which occurred either synchronously with, or between 3 and 18 months before, the clinical detection of relapse.

From Boston, Sorokin et al. (1974) document a prospective study of 102 patients after 'curative' resection of large bowel cancer. Six had a progressive rise in C.E.A. titre above $2.5 \mathrm{ng} / \mathrm{ml}$ and all developed clinical recurrence from 0 to 29 months after this finding. A further twelve patients with transient or stable elevation of titre and eighty-four with levels constantly under $2.5 \mathrm{ng} / \mathrm{ml}$ remained free from recurrence, although two patients developed a second primary. The prognosis for recurrent large bowel cancer is not always hopeless and it is conceivable that routine C.E.A. estimation will be of value in detecting tumour recurrence at a time when further radical surgery is possible. We have recently reviewed previous publications in this field (Ellis, 1974) and these, even without the benefit of modern screening techniques, give grounds for some optimism. In our own series of thirty-nine 'second look' operations, three patients developed entirely new cancers in other organs, eleven developed metachronous tumours in another part of the large bowel and were submitted to resection, four had local recurrence which was only suitable $f \circ r$ palliative surgery, six had recurrences elsewhere which were resected, four had disseminated deposits and there were three patients who were found to have entirely non-malignant conditions with no evidence of recurrence. In this small series, twelve of the thirtynine patients achieved good long term results with survival of 2 years or more and eight of these are still alive. Obviously these figures could be improved still further by sub-clinical detection of recurrent disease.

A further vista opened by these studies on plasma C.E.A. is the possibility that the institution of chemotherapy or radiotherapy at an early phase, when there is only laboratory evidence of recurrence or metastasis, may improve survival. Naturally, this could only be determined by a carefully planned trial.

It might be hoped that a raised plasma C.E.A. in patients with ulcerative colitis might act as a useful warning that malignant change is threatened or has actually occurred, possibly at subclinical level. The studies of Dilawari et al. (1974) have been rather disappointing in this respect. A series of 152 patients with ulcerative colitis was studied, including seven with severe dysplastic changes and six with established carcinoma. C.E.A. levels above $12.5 \mathrm{ng} / \mathrm{ml}$ were found in fifty-three of the patients, but this level was also detected in nearly $50 \%$ of the control series with non-inflammatory bowel disease or with minor anal conditions. Only two of the 152 patients (one in the dysplastic and one in the carcinoma group) had levels above $40 \mathrm{ng} / \mathrm{ml}$, a level usually associated with carcinoma. These authors suggest that C.E.A. levels do not contribute to the management of patients with colitis.

\section{Healing of anastomoses}

Failure of healing of a gastro-intestinal anastomosis with consequent leakage, spreading peritonitis, local abscess or bowel fistula is a complication which the abdominal surgeon has good cause to fear; not only does it put his patient's life in jeopardy but it 
also reflects on his technical skill. Certain practical points have been learned in the bitter school of practical experience; anastomoses at either extremity of the alimentary canal-the oesophagus or the rectum-are the most likely ones to give problems, whereas those of the small bowel and stomach usually heal well. Moreover, an anastomosis carried out in the face of large bowel obstruction or peritonitis is fraught with danger compared with comparative safety in resections of obstructed small intestine (Leading article, British Medical Journal, 1974).

In a recent review of this subject, Everett (1974) stresses three considerations: the blood supply to the ends of the resected bowel, adherence of surrounding structures, and the technique of the anastomosis. An adequate blood supply is essential and gross leakage is probably most often due to inadequate vascularity of the cut ends of the intestine. It is essential that the surgeon ensures a brisk local circulation before he attempts to join the ends of gut. Adhesions are often regarded as a menace which may produce intestinal obstruction, yet we were able to show experimentally that if adhesions are prevented from reaching the bowel whose blood supply is in jeopardy by wrapping it in polythene sheeting, then gangrene inevitably results. Adhesions act, in fact, as vascular ingrowths into the damaged tissue which is always present at the anastomotic line and help to maintain circulation during the vital early days of wound healing. There is much to be said for wrapping the anastomosis in the adjacent omentum which forms, in effect, a pedicled vascular graft (Ellis, 1974a, b).

The exact technique of bowel suture is probably far less important than the other local factors. Most surgeons perform a conventional anastomosis in two layers made up of an inner continuous or interrupted layer, through all the coats of the gut wall, and an outer seromuscular layer. Most use non-absorbable material (thread or silk) for the oesophagus and the colon, at least in the outer layer, but absorbable sutures for gastric anastomoses (where non-absorbable sutures may produce stomal ulceration), and for small intestine. There is evidence that the synthetic absorbable suture material made from polyglycolic acid provokes less inflammation than catgut, but its absorption in human wounds is more protracted than the latter more conventional suture material, thus providing more reliable support during the healing process.

Other factors, both local and general, have recently come under critical clinical and experimental study. Stewart, MacNaughton and Thorley (1974) at Westminster Hospital have shown that anastomotic healing in the experimental model is depressed by the presence of either local or distant malignant disease, and the latter is related, at least in part, to the associated lower plasma protein level. Radiotherapy may seriously impair healing; thus Wellwood, Jackson and Bates (1974) report five patients who had no less than eleven instances of small bowel anastomotic breakdown (all fatal) after radiotherapy to the pelvis. This situation is particularly likely to occur when intra-abdominal adhesions have tethered a loop of small bowel in the pelvis and where heavy irradiation of this segment cannot, therefore, be avoided. They advise that resection in such cases should be carried out widely to avoid the damaged area and if further breakdown occurs it is safer to exteriorize the two ends of the bowel rather than to risk further resection.

Clinical studies have shown that bloody or traumatic colonic and rectal operations are associated with a high incidence of anastomotic dehiscence and it has been suggested that trauma itself has important systemic effects on colonic healing. Irvin and Hunt (1974a) studied the effects of local and remote trauma in colonic anastomoses in rats. Remote trauma (fracture of the femur) had no effect on the tensile and collagen content of the anastomoses, but local trauma to soft tissues near the suture line resulted in loss of colonic tensile strength and a significant incidence of anastomotic breakdown. This did not occur when the intra-abdominal trauma was carried out away from the area of the anastomosis. These authors, therefore, conclude that sepsis, facilitated by local tissue injury and bleeding, rather than the effects of trauma itself, is the important factor. In further experiments (Irvin and Hunt, 1974b) these workers showed that a preliminary colostomy, fashioned 1 month before the anastomosis, or the use of intra-peritoneal cephalothin, abolished the malign effects of local trauma on anastomotic disruption, thus supporting their thesis that sepsis is the immediate cause of anastomotic breakdown in the presence of local tissue trauma. In our own studies, intra-muscular sterile abscesses produced by turpentine injection, bacterial intramuscular abscesses produced by Pseudomonas aeruginosa and transient bacteraemia with the same organisms were all found to have a marked inhibitory effect on the healing of anastomoses in the stomach wall of the rat as well as impairing the healing of the skin, muscle and peritoneum (de Haan, Ellis and Wilks, 1974).

Among other useful contributions in the field of wound healing this year, we may mention the interesting study by Rahmat, Norman and Smith (1974) on the effects of zinc deficiency (which may occur clinically in patients of long term intravenous feeding) on wound healing. They found that rats on a zinc-deficient diet had decreased tensile strength of superficial incisions compared with controlled animals and those on a zinc supplemented diet, 
although the histological and electron microscopic appearances were unaffected. An important finding was that the zinc-deficient animals lose weight, and it might be that zinc deficiency may produce this impairment of healing as a result of the accompanying anorexia. Another possibility is that zincdependent enzymes may be involved in the healing process.

Surgeons always dislike operating on patients on long-term steroids; Engquist, Backer and Jarnum (1974) have studied 100 patients on steroid treatment (including eighty-four on permanent steroid therapy) compared with 100 matched controls subjected to surgery. The only significant increase in complication rate concerned wound infection which occurred in eleven of the patients in the steroid group compared with only one in the controls. Since infection may predispose to anastomotic breakdown, these findings confirm our distaste at performing surgery in this group of patients.

\section{Burns}

In spite of considerable advances, no one can deny that major burns still represent a challenging problem. Over the last 30 years, the fluid and electrolyte loss into the burned area has come to be well understood, and early and adequate therapy has practically eliminated death from shock in the early days after a severe burn. Yet the mortality remains high, especially in children and even more so in elderly patients. Sepsis due to infection of the wound appears as the most obvious cause for this, yet it is disappointing that systemic and local antibiotics together with immunotherapy have not had the beneficial effect that might have been expected of them, and this failure is especially seen in the elderly patient. Is it because the wrong antibiotic combinations are being used? Hartford et al. (1974) studied two groups of patients with more than $10 \%$ of body surface burnt. Both groups received the same local treatment with $0.5 \%$ silver nitrate dressings. In the first series of 236 patients, treated up to 1970, a 5-day course of streptomycin and penicillin was given, followed by systemic antibiotics if and when indicated. There were sixty-one deaths in this group, $62 \%$ being due to bacterial infection. From 1970 onwards a further 227 patients were treated. All were given penicillin but if $30 \%$ or more of the body surface was burnt polymyxin and/or kanamycin was prescribed. If renal failure was present chloramphenicol and/or carbenicillin was used. In this series there were forty-six deaths, $35 \%$ being due to infection. A clinical study of these two groups showed that it was only in patients under the age of 40 with burns of $30-59 \%$ of the body surface where there was a statistically significant decrence in mortality from $19 \%$ to $1 \%$. These authors also stress that adjustments in antibiotic therapy must be made depending on the bacterial population and their sensitivities in the Burns Unit.

The special hazard of the extensive burn has led to the postulation that a burn toxin is produced which is responsible for many of the malign effects of the extensive full-thickness burn. Cuevas et al. (1974) put forward evidence that a vasoactive substance produced from the burnt tissue mobilizes endotoxin from the gastrointestinal tract. They demonstrated that rabbits subjected to extensive immersive burns develop endotoxaemia in $2 \mathrm{hr}$, with severe plasma volume deficit, vascular collapse and death in from 10-12 hr. The liver and blood of these animals contain an endotoxin and a Gram-negative bacteraemia can be demonstrated. This state of affairs is prevented by fluid and electrolyte therapy, together with local sulfamylon and systemic kanamycin. A fatal endotoxaemia was produced by giving rabbit's blood obtained from the venous drainage of the burnt area taken at the stage before it contained endotoxin. The nature of this vasoactive substance remains to be determined, but its effects are similar to those obtained by slow intravenous infusion of serotonin, bradykinin, noradrenaline or histamine into normal rabbits. Endotoxaemia did not occur in rabbits whose intestine was free from Gram-negative organisms. From Switzerland, Allgower, Stadtler and Schoenberger (1974) have reported fascinating studies on burn toxin; they bring forward evidence that this toxin is derived from a thermal polymerization of cell membrane lipoproteins within the dermis. Using ultracentrifugation, they have identified this substance in burnt skin in the mouse and in the human and have also identified the same material in the serum from extensively burnt patients. Active immunization of mice with sublethal doses of human toxin reduced mortality of extensive burns from $100 \%$ in controls to $60 \%$ in immunized animals; furthermore, passive immunotherapy with the antiburn IgG also proved very effective, leading to a permanent survival of $80 \%$ of the animals subjected to a burn which would have otherwise always been fatal. Three groups of mice were employed in a further study; groups 1 and 2 were pre-injected with burn toxin while the 3rd group, the controls, received saline. After $72 \mathrm{hr}$ all three groups were inoculated into a subcutaneous wound with Pseudomonas sp. in a standard dosage. In addition, group 2 was treated at this same time with anti-toxic IgG. In the first group the combined effect of toxin and subsequent infection produced a mortality of $80 \%$ compared with $20 \%$ for the controls. In group 2, however, the injection of antitoxic IgG in addition to the toxin had the effect of reducing mortality to the same level as in the controls. Mortality from Pseudomonas infection was not 
increased by the previous injection of material from unburned animals. These experiments indicate that a specific burn toxin, most probably produced by generalized damage to cell wall membranes, does enhance the establishment of septicaemia. Infection may be crucial in borderline cases and it may be that passive immunotherapy may have an important part to play in the management of extensive burns.

The early treatment of the burnt area has now reached a stage of high efficiency. Perhaps the most important recent advance in management has been the development of silver sulphadazine as a local dressing, since this has a good spectrum of activity and does not cause appreciable pain or electrolyte disturbance when applied. Circumferential burns can be treated with the exposure technique using a support system resembling an upturned hovercraft, termed a hoverbed. The patient may be covered with a radiant heat-reflecting surface which floats above him on the current of air and he is thus enclosed in a sterile, warm micro-climate (Sanders, 1974). Most surgeons perform excision of full thickness burns at about the 14th day, skin grafting usually being performed at the same operation (Evans, 1974). Biological dressings such as freeze-dried cadaver skin, pig skin or amnion, have helped to cover denuded surfaces until they can be replaced with autografts. Hackett and Bowen (1974) have compared lyophilized (freeze-dried) cadaver skin allografts with lyophilized xenografts in the form of irradiationsterilized porcine skin which has now become available commercially. Both appear to be equally effective for the temporary cover of raw areas. Lyophilized allograft has the disadvantage of occasionally separating into two layers when removed; the deeper layer can easily be missed and may inhibit the take of the subsequent autograft. The xenograft is easier to handle and, since it is commercially produced, should become more easily available.

One of the great disappointments of burn treatment is the development of hypertrophic scarring and contracture, which is particularly seen in children. Although clinically a nonhypertrophic scar may appear to be inactive, the rate of collagen synthesis in such a scar is nearly double that of normal skin. In hypertrophic scar the rate of collagen production is over four times that of normal skin and in keloids this increases to some thirteen times normal. In nonhypertrophic scar, the rate of collagen synthesis is in equilibrium with the rate of collagen breakdown, but in hypertrophic scar this balance no longer exists because of this excessive production. Histochemical studies by Harvey Kemble and Brown (1974) indicate that there is an increase in keratin synthesis and in the degradation of fats, proteins and mucopolysaccharides, but there seems to be little change in the metabolism of glycogen and nucleo-proteins. Obviously the analysis of the abnormal metabolic processes which produce hypertrophic scars is a prerequisite to the rational formulation of treatment.

An important advance in the management of hypertrophic scarring is the use of compression treatment, which is effective both as a prophylactic and a therapeutic method. Naturally, it is difficult to maintain continued compression of a burnt area, but this has been greatly aided by the introduction of Prenyl, which is an unvulcanized neoprene. This material is malleable at $60^{\circ} \mathrm{C}$ and may be stretched and moulded to the body contours. It is bandaged firmly in place and the splint then hardens and retains an elastic moulded shape. It is worn continuously but is removed once daily and washed in cold water before re-applying. Thomson (1974) reports the use of this occlusion treatment in 100 consecutive patients with hypertrophic scarring; seventy-two of these responded and the majority were improved both cosmetically and functionally. The remaining twenty-eight patients either defaulted from treatment or developed early or late allergic reactions. Two of the patients showed no response at all because the scars were too old and mature. The mode of action of compression on hypertrophic scar is not known, but may be produced partly by reduction of the oedema fluid and also by some influence on the cross-linkage of collagen. This fascinating topic requires further study.

\section{Miscellanea}

A number of interesting and eye-catching papers deserve individual comment. Clinicians in this country tend to regard tuberculosis today as a rare disease, but there is no doubt that, particularly in the big cities, this is far from true and must be especially considered in immigrant patients. Iles and Emerson (1974) report thirty-two adult patients with tuberculous lymphadenitis, fourteen of whom were from abroad. In twelve instances the affected lymphnode was excised without chemotherapy and ten of these patients relapsed. Local excision, together with anti-tuberculous chemotherapy with at least two drugs, was followed by no instances of relapse. Of the fourteen mycobacteria which were cultured, thirteen proved to be human strain organisms. The authors advise triple therapy with streptomycin, isoniazid and rifampicin which should be started immediately after operation and continued until the results of drug sensitivities are known in about 6 weeks. The streptomycin can then, if appropriate, be discontinued and 2-drug chemotherapy then continued for 2 years. If nodes continue to enlarge or become fluctuant during treatment, further excision should be carried out.

An intriguing paper entitled 'The Cast Syndrome 
Incognito' (Hall, 1974) points out that acute gastric dilatation following the application of a body or hip spica plaster-cast is well recognized; acute lordosis results in obstruction of the third and fourth parts of the duodenum between the superior mesenteric vessels anteriorly and the lumbar spine behind. However, Hall points out that the syndrome may occur in the absence of a plaster-cast. He reports four patients placed on lower limb or skull traction for multiple injuries, all of whom developed projectile vomiting. All were asthenic persons who displayed a moderate rapid weight loss immediately after the accident and all of whom were nursed in an immobile supine position. The diagnosis was confirmed in each case by barium studies, which demonstrated gastric dilatation, dilatation of the second and third portions of the duodenum and a sharp cut-off at the level of the superior mesenteric artery. All of these patients responded to conservative measures and in each case the orthopaedic treatment had to be changed or modified to deal with this situation. This might involve getting the patient up out of bed with internal fixation of the fractures or the use of a circoelectric bed.

Localized obstruction of the iliac arteries producing the Leriche syndrome has usually indicated major surgical treatment by disobliteration or endarterectomy. A paper by Dotter et al. (1974), from Portland, Oregon, describes an interesting technique in which percutaneous transluminal dilatation of stenosed atheromatous iliac arteries was carried out in forty-three patients under local anaesthesia (forty-eight stenotic vessels). A reinforced balloon catheter was employed, and either step-wise distension used for long strictures or a pull-through technique for short stenoses. Thirty-nine $(81 \%)$ had lasting, if not permanent, improvement up to 75 months after dilatation. Two of four delayed recurrences were successfully retreated. Failure of this treatment does not preclude subsequent surgery. The authors consider that, unlike fibrotic scar tissue, the noncellular material comprising the bulk of the mature atheromatous lesion is capable of retaining an in situ compression remodelling that is achieved by forcing the lesion against the surrounding outer sheath of arterial wall.

Perhaps the most unusual syndrome to be described this year is 'Bullman's hand', a case of which is reported by O'Sullivan and Brady (1974) from Cork. In rural Ireland the colloquial term for an artificial inseminator is 'the Bullman'. The technique employed is for the operator to insert the left hand and arm, encased in a long rubber glove and sleeve, into the cow's rectum to grasp the cervix. The inseminating pipette is inserted through the cervix with the other hand. A busy artificial inseminator will perform this manoeuvre as often as thirty times a day. In addition to the constricting effect of the rubber glove and sleeve, the tone of the cow's anal sphincter is considerable on the upper forearm of the operator. When to this is added the cold damp surroundings, together with the forced flexion of the index and middle fingers against resistance, it is not surprising that digital ischaemia may be precipitated by this occupation. These authors report such a case, in which brachial angiography showed occlusion of the digital vessels of the radial $2 \frac{1}{2}$ fingers. The patient's symptoms were greatly relieved after he had taken his surgeon's advice to discontinue his occupation and to take up a clerical post.

\section{References}

\section{Complications following cholecystectomy}

Bartlett, M.K., Warshaw, A.L. \& OtTINGer, L.W. (1974) The removal of biliary duct stones. Surgical Clinics of North America, 54, 599.

Bremner, D.N., McCormick, J.S.C., Thomson, J.W.W. \& MCNaIR, T.J. (1974) A study of cholecystectomy. Surgery, Gynecology and Obstetrics, 138, 752.

BurHenNe, H.J. (1974) Nonoperative roentgenologic instrumentation technics of the postoperative biliary tract. Treatment of biliary stricture and retained stones. American Journal of Surgery, 128, 111.

CatT, P.B., Hogg, D.F., Clunie, G.J.A. \& Hardie, I.R. (1974) Retained biliary calculi: removal by a simple nonoperative technique. Annals of Surgery, 180, 247.

Ellis; H. \& ADAIR, H.M. (1974) Bile peritonitis-a report of fifteen patients. Postgraduate Medical Journal, 50, 713.

GLENN, F. (1974) Retained calculi within the biliary duct system. Annals of Surgery, 179, 528.

Halvorsen, J.F. \& SEMB, B.K.H. (1974) Traumatic false aneurysm of the right hepatic artery with rupture into the duodenum: an unusual complication of biliary surgery. Acta chirurgica scandinavica, 140, 252.

Keighley, M.R.B., Lister, D.M., JACOBS, S.I. \& Giles, G.R. (1974) Hazards of surgical treatment due to micro organisms in the bile. Surgery, 75, 578.

Lahana, D.A., BonorRis, G.G. \& Schoenfield, L.J. (1974) Gallstone dissolution in vitro by bile acids, heparin, and quaternary amines. Surgery, Gynecology and Obstetrics, 138, 683.

Lansford, C., Mehta, S. \& Kern, F. (1974) The treatment of retained stones in the common bile duct with sodium cholate infusion. Gut, 15, 48.

PeEl, A.L.G. \& Ritchie, H.D. (1974) Gilbert's syndrome in patients with gallbladder stones. Annals of the Royal College of Surgeons of England, 55, 184.

RoMero, R. \& BUTterfield, W.C. (1974) Heparin and gallstones. American Journal of Surgery, 127, 687.

Semb, B.K.H., Norderval, Y. \& Halvorsen, J.F. (1974) The non-operative removal of retained common duct stones after biliary surgery. Acta chirurgica scandinavica, 140, 469.

WarSHAW, A.L. \& Bartlett, M.K. (1974) Technic for finding and removing stones from intrahepatic bile ducts. American Journal of Surgery, 127, 353.

WAY, L.W. (1973) Retained common bile duct stones. Surgical Clinics of North America, 53, 1139.

\section{Acute pancreatitis}

Feldstein, J.D., Johnson, F.R., Kallick, C.A. \& Doolas, A. (1974) Acute hemorrhagic pancreatitis and pseudocyst due to mumps. Annals of Surgery, 180, 85. 
Feller, J.H., Brown, R.A., Toussaint, G.P.M. \& ThompSON, A.G. (1974) Changing methods in the treatment of severe pancreatitis. American Journal of Surgery, 127, 196.

Francillon, J., Vignal, J., Grandjean, J.P., Tissot, E. \& BAYLE, E. (1974) Acute necrosing pancreatitis. With a report of 14 cases. Chirurgia gastroenterologica, 8, 53 .

Hallberg, D. \& Theve, N.O. (1974) Observations during treatment of acute pancreatitis with insulin and glucose infusion. Acta chirurgica scandinavica, 140, 138.

IMRIE, C.W. (1974a) Observations on acute pancreatitis. British Journal of Surgery, 61, 539.

IMRIE, C.W. (1974b) Facial flushing in acute pancreatitis. British Medical Journal, iv, 593.

ImRIe, C.W., Whyte, A.S. \& Blumgart, L.H. (1974) A prospective survey of the treatment of acute pancreatitis. Gut $, 15,340$.

Leading Article (1974) Trasylol for pancreatitis. British Medical Journal, iii, 133.

Norton, L. \& EISEMAN, R. (1974) Near total pancreatectomy for hemorrhagic pancreatitis. American Journal of Surgery, 127, 191

Sankaran, S., Lucas, C.E. \& Walt, A.J. (1974) Transient hypertension with acute pancreatitis. Surgery, Gynecology and Obstetrics, 138, 235.

Trapnell, J.E. (1974) Acute pancreatitis. British Journal of Hospital Medicine, 12, 193.

Trapnell, J.E., Rigby, C.C., Talbot, C.H. \& Duncan, E.H.L. (1974) A controlled trial of Trasylol in the treatment of acute pancreatitis. British Journal of Surgery, 61, 177.

Welbourn, R.B. \& Cox, A.G. (1974) Glucagon therapy in acute pancreatitis. British Medical Journal, i, 244.

WYATT, A.P. (1974) Diagnosis and management of acute pancreatitis. Annals of the Royal College of Surgeons of England, 54, 229.

\section{Renal calculi}

BLANDY, J.P. (1974) Urinary calculus. British Journal of Urology, 46, 117.

LeONG, C.H., YU, H. \& ONG, G.B. (1974) Emergency surgery for calculus diseases of the upper urinary tract. British Journal of Surgery, 61, 410.

Marshall, V. \& Blandy, J. (1974) Simple renal hypothermia. British Journal of Urology, 46, 253.

MORRISON, R.B.I. (1974) Metabolic aspects of urinary calculi in Wellington. British Journal of Urology, 46, 117.

Powis, S.J.A., Black, J., Macdougall, J.A. \& Clews, J.W. (1974) Management of patients with urinary calculi. British Medical Journal, i, 355.

SReenevasan, G. (1974) Bilateral renal calculi. Annals of the Royal College of Surgeons of England, 55, 3.

Sutor, D.J., Wooley, S.E. \& Illingworth, J.J. (1974) Some aspects of the adult urinary stone problem in Great Britain and Northern Ireland. British Journal of Urology, 46, 275.

Walsh, A. (1974) An aggressive approach to stones in the lower ureter. British Journal of Urology, 46, 11.

\section{Venous thrombosis and pulmonary embolism}

Browse, N.L. (1974a) Thrombosis: treatment and prophylaxis. British Medical Journal, iv, 96.

BrowSE, N.L. (1974b) Current thoughts on venous thromboembolism. Surgical Clinics of North America, 54, 229.

Browse, N.L., Jackson, B.T., Mayo, M.E. \& Negus, D. (1974) The value of mechanical methods of preventing postoperative calf vein thrombosis. British Journal of Surgery, 61, 219.

Carter, A.E. \& Eban, R. (1974) Prevention of postoperative deep venous thrombosis in legs by orally administered hydroxychloroquine sulphate. British Medical Journal, iii, 94.
Clark, W.B., Macgregor, A.B., Prescott, R.J. \& Ruckley, C.V. (1974) Pneumatic compression of the calf and postoperative deep-vein thrombosis. Lancet, ii, 5.

Cunningham, I.G.E. \& Young, N.K. (1974) The incidence of deep-vein thrombosis in Malaysia. British Journal of Surgery, 61, 482.

Doran, F.S.A. (1974) Use of the electronic gaiter. Proceedings of the Royal Society of Medicine, 67, 705.

EBERT, P.A. (1974) The role of surgery in the treatment of pulmonary thromboembolism. Surgical Clinics of North America, 54, 1107.

Fossard, D.P., Friend, J.R., Field, E.S., Corrigan, T.P., Kakkar, V.V. \& Flute, P.T. (1974a) Fibrinolytic activity and postoperative deep-vein thrombosis. Lancet, i, 9.

Fossard, D.P., Kakkar, V.V., Corrigan, T.P. \& Srachan, C.J.L. (1974b) The origin of deep-vein thrombosis-a phlebographic study. British Journal of Surgery, 61, 332.

Gardner, A.M.N., AsKew, A.R., HARSe, H.R., WilmsHURST, C.C. \& TuRner, M.J. (1974) Partial occlusion of the inferior vena cava in the prevention of fatal pulmonary embolism. Surgery, Gynecology and Obstetrics, 138, 17.

GoRDON-SMITH, I.C. (1974) Nonmechanical methods of prevention. Proceedings of the Royal Society of Medicine, 67, 704.

Hassan, M.A., Rahman, E.A. \& Rahman, I.A. (1974) Prostatectomy and deep-vein thrombosis in Sudanese patients. British Journal of Surgery, 61, 650.

JofFE, S.N. (1974) Racial incidence of postoperative deepvein thrombosis in South Africa. British Journal of Surgery, $61,982$.

KAKKAR, V.V. (1974) Review of present status of prophylaxis of venous thromboembolism. Proceedings of the Royal Society of Medicine, 67, 706.

Knight, L., Amplatz, K. \& Nicoloff, D.M. (1974) Alter nate method for introduction of inferior vena cava filter. Surgery, Gynecology and Obstetrics, 138, 763.

Lahnborg, G., Bergstrom, K., Friman, L. \& Lagergren, H. (1974) Effect of low-dose heparin on incidence of postoperative pulmonary embolism detected by photoscanning. Lancet, i, 329.

MARION, P. \& BINET, J.P. (1974) Embolies pulmonaires. Journal de chirurgie, Paris, 108, 135.

Myhre, H.O., Storen, E.J. \& Ongre, A. (1974) The incidence of deep-venous thrombosis in patients with leg oedema after arterial reconstruction. Scandinavian Journal of Thoracic and Cardiovascular Surgery, 8, 73.

Negus, D. \& RiCKForD, C.R.K. (1974) Practical problems in diagnosis. Proceedings of the Royal Society of Medicine, 67, 703 .

Roberts, V.C. \& Cotton, L.T. (1974) Prevention of postoperative deep-vein thrombosis in patients with malignant disease. British Medical Journal, i, 358.

Silver, D. (1974) Pulmonary embolism. Prevention, detection and nonoperative management. Surgical Clinics of North America, 54, 1089.

Skillman, J.J. (1974) Postoperative deep-vein thrombosis and pulmonary embolism: a selective review and personal viewpoint. Surgery, 75, 114.

Tibbutt, D.A., Davies, J.A., Anderson, J.A., Fletcher, E.W.L., Hamill, J., Holt, J.M., Lea Thomas, M., Lee, G. DE J., Miller, G.A.H., Sharp, A.A. \& Sutton, G.C. (1974) Comparison by controlled clinical trial of streptokinase and heparin in treatment of life-threatening pulmonary embolism. British Medical Journal, i, 343.

TibiutT, D.A. \& GunNing, A.J. (1974) Calf haematoma: a new sign in differential diagnosis from deep-vein thrombosis. British Medical Journal, iv, 204.

Van Vroonhoven, T.J.M.V., Van ZiJl, J. \& Muller, H. (1974) Low-dose subcutaneous heparin versus oral anticoagulants in the prevention of postoperative deep-venous thrombosis. Lancet, i, 375. 


\section{Growing points in cancer}

Booth, S.N., Jamieson, G.C., King, J.P.G., Leonard, J., OATES, G.D. \& DyKes, P.W. (1974) Carcinoembryonic antigen in management of colorectal carcinoma. British Medical Journal, iv, 183.

CAstro, J.E. \& CASs, W. (1974) Maintenance of human tumours and tissues in immunosuppressed mice. British Journal of Surgery, 61, 421.

Dilawari, J.B., Lennard-Jones, J.E., MaCkay, A.M., Ritchie, J.K. \& SturzaKer, H.G. (1974) C.E.A. estimation in ulcerative colitis with and without malignant change. Gut, 15, 828.

Ellis, H. (1974) 'Second-look surgery' for suspected recurrences in cancer of the large bowel. Cancer Treatment Reviews, 1, 205.

Feneley, R.C.L., Eckert, H., Riddell, A.G., Symes, M.O. \& TRIBE, C.R. (1974) The treatment of advanced bladder cancer with sensitized pig lymphocytes. British Journal of Surgery, 61, 825.

Kelleher, J., Colum, S., Doyle, C., Hennessy, T. \& Whelton, M.J. (1974) Alpha fetoprotein in metastatic gastric carcinoma. Gut, 15, 401 .

KEw, M. (1974) Alpha fetoprotein in primary liver cancer and other diseases. Gut, 15, 814.

KoHN, J. \& Weaver, P.C. (1974) Serum-alpha-fetoprotein in hepatocellular carcinoma. Lancet, ii, 334.

LeADing ARTICle (1974a) Vinyl chloride and cancer. British Medical Journal, i, 590.

LeAding ARTICLE (1974b) More facts on vinyl chloride and cancer. British Medical Journal, iv, 486.

LEADING ARTICLE (1974c) Human tumour xenografts. British Medical Journal, iv, 366.

Leading Article (1974) Vinyl chloride, P.V.C., and cancer. Lancet, i, 1323.

LeE, F.I. \& HARRY, D.S. (1974) Angiosarcoma of the liver in a vinyl-chloride worker. Lancet, $\mathbf{i}, 1316$.

Mackay, A.M., Patel, S., Carter, S., Stevens, U., LawRENCE, D.J.R., CoOPER, E.H. \& Neville, A.M. (1974) Role of serial plasma C.E.A. assays in detection of recurrent and metastatic colorectal cancers. British Medical Journal, iv, 382.

MaGEe, P.N. (1974) Carcinogens in the environment. Proceedings of the Royal Society of Medicine, 67, 741.

Sorokin, J.J., Sugarbaker, P.H., Zamcheck, N., Pisick, M., KuPChIK, H.Z. \& Moore, F.D. (1974) Serial carcinoembryonic antigen assays. Use in detection of cancer recurrence. Journal of the American Medical Association, 228, 49.

\section{Healing of anastomoses}

De HaAN, B.B., Ellis, H. \& Wilks, M. (1974) The role of infection on wound healing. Surgery, Gynecology and Obstetrics, 138, 693.

Ellis, H. (1974a) Intraperitoneal adhesions. British Journal of Hospital Medicine, 11, 401.

Ellis, H. (1974b) Post-operative Peritoneal Adhesions. In: Surgery lectures Vol. 2 (Ed. by J. McFarland). London, Butterworths.

ENGQuist, A., BACKer, O.G. \& JARnum, S. (1974) Incidence of post-operative complications in patients subjected to surgery under steroid cover. Acta chirurgica scandinavica, 140,343 .
EVERETT, W.G. (1974) Sutures, incisions and anastomoses. Annals of the Royal College of Surgeons of England, 55, 31.

IRVIN, T.T. \& HUNT, T.K. (1974a) The effect of trauma on colonic healing. British Journal of Surgery, 61, 430.

IRvin, T.T. \& HunT, T.K. (1974b) Pathogenesis and prevention of disruption of colonic anastomoses in traumatised rats. British Journal of Surgery, 61, 437.

LeADING ARTICle (1974) Anastomotic leakage. British Medical Journal, iv, 251.

Rahmat, A., Norman, J.N. \& Smith, G. (1974) The effect of zinc deficiency on wound healing. British Journal of Surgery, 61, 271.

Stewart, R.J., Macnaughton, J.I. \& Thorley, R.S. (1974) Experimental observations on intestinal healing in malignant disease. Annals of the Royal College of Surgeons of England, 54, 269.

Wellwood, J.M., JACkson, B.T. \& BATES, T.D. (1974) Breakdown of small-bowel anastomoses after pelvic radiotherapy. Annals of the Royal College of Surgeons of England, 54, 306.

\section{Burns}

Allgower, M., Stadtler, K. \& Schoenberger, G.A. (1974) Burn sepsis and burn toxin. Annals of the Royal College of Surgeons of England, 55, 226.

Cuevas, P., IshiYama, M., Koizumi, S., Woodruff, P., KAUfMAN, A. \& Fine, J. (1974) Role of endotoxemia of intestinal origin in early death from large burns. Surgery, Gynecology and Obstetrics, 138, 725.

Evans, A.J. (1974) Surgical aspects of burns. Proceedings of the Royal Society of Medicine, 67, 253.

Hackett, M. \& Bowen, J. (1974) Preliminary report on the comparative use of lyophilized homograft and xenograft in the closure of raw areas. British Journal of Surgery, 61, 427.

Hartford, C.E., Budensteiner, J.A., Boyd, W.C. \& ZIFFREN, S.E. (1974) The use of multiple systemic antibiotics in the treatment of patients with burns. Surgery, Gynecology and Obstetrics, 138, 837.

Harvey Kemble, J.V. \& Brown, R.F.R. (1974) Histochemistry of hypertrophic scars and keloids. Proceedings of the Royal Society of Medicine, 67, 257.

SANDERS, R. (1974) The burnt patient: a general view. British Medical Journal, iii, 460.

Thomson, W.G. (1974) Treatment of hypertrophic scarring by compression and occlusion. Proceedings of the Royal Society of Medicine, 67, 256.

\section{Miscellanea}

Dotter, C.T., Rosch, J., Anderson, J.M., Antonovic, R. \& Robinson, M. (1974) Transluminal iliac artery dilatation. Nonsurgical catheter treatment of atheromatous narrowing. Journal of the American Medical Association, 230, 117.

Hall, L.W. (1974) The cast syndrome incognito. American Journal of Surgery, 127, 317.

Iles, P.B. \& EMERSON, P.A. (1974) Tuberculosis lymphadenitis. British Medical Journal, i, 143.

O'Sullivan, D.J. \& BRADY, M.J. (1974) Bullman's hand-an unusual occupational lesion. Journal of the Irish Medical Association, 67, 102. 